\title{
TRACKING THE SPATIAL EVOLUTION OF URBAN HEAT ISLANDS
}

\author{
Rui Zhu ${ }^{\mathrm{a}, *}$ Eric Guilbert ${ }^{\mathrm{b}}$, Man Sing Wong ${ }^{\mathrm{c}}$ \\ ${ }^{a}$ Dept. of Land Surveying and Geo-informatics, The Hong Kong Polytechnic University, Hong Kong, China - r.zhu@ connect.polyu.hk \\ ${ }^{\mathrm{b}}$ Dept. of Geomatics Sciences, Laval University, Québec, G1V 0A6 (QC) Canada - eric.guilbert@scg.ulaval.ca \\ ${ }^{c}$ Dept. of Land Surveying and Geo-informatics, The Hong Kong Polytechnic University, Hong Kong, China - 1s.charles@ polyu.edu.hk
}

Commission II, WG II/1

KEY WORDS: Spatiotemporal data modeling; Urban heat islands; Remote sensing

\begin{abstract}
:
The urban heat island (UHI) phenomenon occurring in the urban areas or city-clusters is increasingly becoming a severe problem in the urbanization process. Previous research mainly rely on grid analysis techniques to study temperature data from images recorded at fixed time instants. The evolutionary process of UHI in both time and space has not been investigated yet. This research designs an object-oriented spatiotemporal model to reconstruct the evolution of UHI and provide a qualitative interpretation. Each UHI is modeled as a spatiotemporal field object with it own life cycle. Dynamic behavior of an UHI is defined by sequences of spatial changes (e.g. contraction or expansion) and topological transformations (e.g. merge or split). The model is implemented in an object-relational database and applied to air temperature data collected from weather stations every hour over three days. UHIs with their behavior were extracted from the data. Results suggest that the model can effectively track and provide a qualitative description of the UHI evolution.
\end{abstract}

\section{INTRODUCTION}

Urban heat island is a phenomenon where temperature in urban areas is obviously higher than in surrounding rural areas. With the increasing rate of urbanization process, many rural areas have gradually become urbanized areas, small and middle-sized cities expand to metropolises, and mega cities grow to spatial contiguous city clusters, which cause the UHIs occurring in the cities or city clusters to have a measurable influence on the weather and even regional climate evolution. Hence, much work has been done to study the causative factors and adverse effects of UHI from thermal intensity images derived from satellite images and from meteorological station records.

Work in this direction consisted mainly in correlating thermal intensity from static surface temperature images with environmental (Lo et al. 1997, Dousset and Gourmelon 2003, Stathopoulou and Cartalis 2006) or social indicators (Buyantuyev and $\mathrm{Wu}$ 2010). Recently, consideration has also been given to studying the dynamic evolution of UHI where UHI are defined as clusters of pixels, moving towards object-based analysis. For example, (Keramitsoglou et al. 2011) proposed an object-based image analysis to reveal thermal pattern that thermal intensities of hotspots are strongly correlated to their extent.

In another direction, GIS tools can be used to study and visualize UHI causative factors such as ventilation (Wong and Nichol 2013). However conventional GIS data models and analytical tools provide no qualitative information about the processes occurring within UHIs and trends that can take place over long periods of time (McIntosh and Yuan 2005). More in-depth study of UHI could be performed by automatically extracting the dynamics of a UHI during its whole lifetime through the analysis of a set of images. Such an approach requires the inclusion of the temporal dimension and thus the extension of the 2D spatial model to a spatiotemporal model. A fully object-oriented model where each UHI is addressed as an object with its own attributes would also help in adding a qualitative description to the information extracted from the data.

\footnotetext{
*Corresponding author
}

Besides works from the remote sensing community, the GIS community has looked at data models to represent dynamic geographical phenomena. The objective of these models is to be able to describe the spatiotemporal behavior of objects observed in sequences of images. Geographical phenomena can be defined as field objects corresponding to geo-objects with an internal structure defined by variations of field-like properties within the object boundary (Goodchild et al. 2007). Their dynamic is driven by their activities, events and processes and can be observed through changes and movements (Yuan and Hornsby 2008). Such an approach fits especially well with environmental phenomena such as UHI which can be seen as objects whose characteristics change through time. Processes and events are spatiotemporal objects defined with time attributes (e.g. starting and ending time), thematic attributes and dynamic attributes describing movement (McIntosh and Yuan 2005) and are linked to entities involved in the events. The data model can combine both raster and vector representations as thematic data are computed from grid points inside the entities (Bothwell and Yuan 2010).

Topological transitions between two consecutive time steps are defined by topological relationships between objects. (Renolen 2000) introduced a framework where each spatiotemporal object experiences its generic behavior as either alive or dead, and seven types of transition between two status: creation, alteration, destruction, reincarnation, annexation, deduction, and reallocation. Several studies applied this framework in specific applications (Bothwell and Yuan 2011 Guilbert and Lin 2007, Nixon and Hornsby 2010) where objects can merge or split leading to the creation and destruction of new objects.

The objective of this study is to provide a new object-oriented model where UHI are seen as spatiotemporal objects with their own thematic and field attributes and their relationships evolving through space and time. The purpose of such a model is to extract spatiotemporal processes UHI go through during their life cycle so as to provide a qualitative description of the UHI behavior. The benefit of the approach is that different types of behaviors can be recorded in a database as UHI properties or relationships in a spatiotemporal database allowing for UHI behavior retrieval 
through simple queries.

The rest of this paper is organized as follows. Section 2 presents related work on spatiotemporal modeling of field data. Section 3 presents the new conceptual model centred on UHI as spatiotemporal objects. Section 4 empirically evaluates the proposed method on a case study. Finally, Section 5 concludes and points to future research.

\section{OBJECT MODELING OF URBAN HEAT ISLANDS}

In our work, UHI are identified from thermal images as regions in urban areas where the temperature is higher than that in rural areas. At this stage, other factors influencing temperature variation are not considered and thermal exchanges at different elevations are omitted. UHI are seen as field objects whose thematic attribute is the temperature intensity defined by the difference with a threshold temperature observed in rural areas at the same time.

With the heat intensity varying through time, UHI appear when the temperature difference is above a threshold and disappear when it falls below this threshold. Hence each UHI has a finite lifetime. While active, the UHI shape can change as it can expand and contract with temperature variation. It can also undergo transformations as neighbouring UHI can overlap or move apart.

In an object-oriented approach, an UHI hence can be modeled as a field-object with spatial (its shape) and thematic (temperature intensity) time-dependent attributes and UHIs can be associated by spatiotemporal relationships. Changes in the UHI shape and structure can be recorded at each time instant to provide a qualitative description of the UHI evolution.

\subsection{UHI changes}

Only movements related to the UHI extent are modeled. From our preliminary observations, we consider that UHI are localized phenomena which do not move away from their point of origin and displacement is not seen significant, even influenced by solar radiation or during the year due to the seasonal changes. At each instant, the UHI movement is defined by one of these three values:

- expanding when the area grows bigger.

- stable when the area remains constant.

- contracting when the UHI is getting smaller.

\subsection{UHI transformations}

Apart from these spatial changes, UHIs go through several transformations during their life cycle. Most obvious transformations are the appearance and disappearance of a UHI when the intensity variation leads to a UHI to appear or to vanish. Other transformations occur when UHIs either split or merge. These operations can be seen in different ways in the UHI life cycle. A merge can be modeled as the disappearance of two UHIs and the appearance of a new one or as the absorption of one by another. Similarly, a split can be seen as the appearance of two new UHIs or as one UHI splitting off another. These transformations are defined as:

- inherited merge when one UHI is absorbed by an existing one;

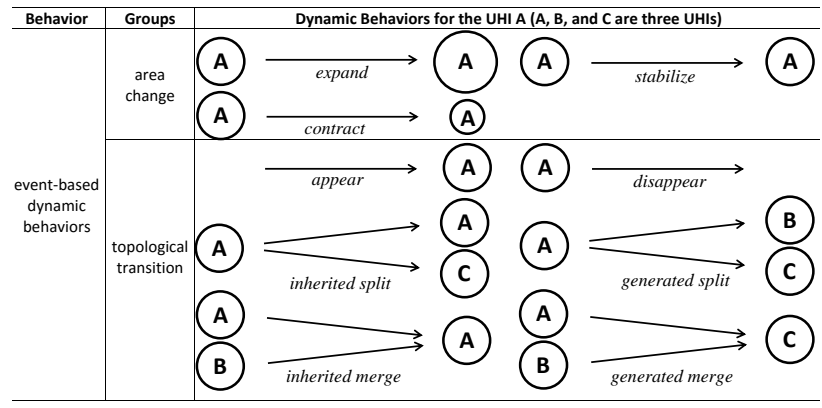

Figure 1: Definition of the dynamic behaviors of an UHI

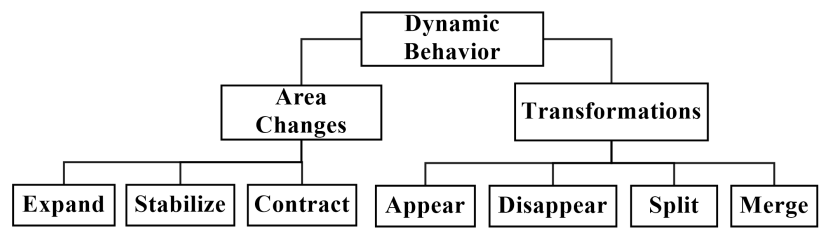

Figure 2: UHI behavior classification

- generated merge when the merge of two UHI leads to a new $\mathrm{UHI}$;

- inherited split when a part of an UHI detaches and forms a new UHI;

- generated split when a split leads to the creation of two new UHI.

All these transformations and changes defined above are illustrated in Figure 1 Changes correspond to continuous transformations which do not modify the UHI structure. As such, they are observed on time intervals. Topological transitions modify the structure as they lead to the creation or removal of UHI. They correspond to discrete operations recorded at a given time instant. Conceptually, all these changes are summarized in a hierarchical set of concepts in Figure 2 The main two categories are movements related to changes in the UHI shape and transformations related to structural changes.

The lifetime of an UHI can then be decomposed in sequences and processes (Figure 3). A sequence is a time interval associated to one type of spatial change. For example a period of time during which an UHI expands corresponds to one sequence. A process occurs at a time instant and relates one or several sequences from one or several UHI with a topological transition. Hence, the lifetime of an UHI can be described by a series of consecutive sequences and by a series of processes.

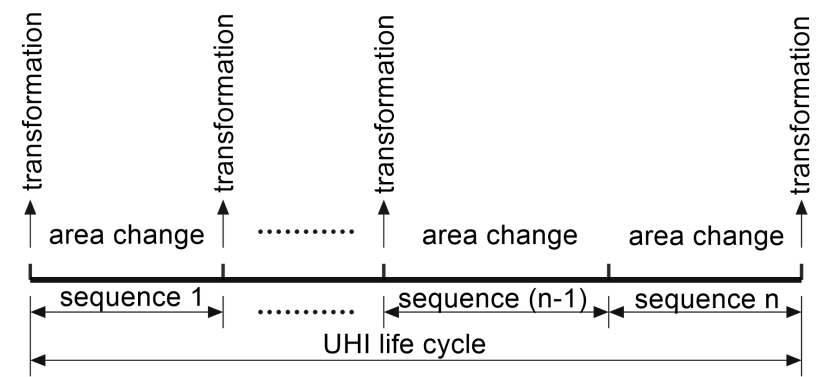

Figure 3: Timeline of an UHI 


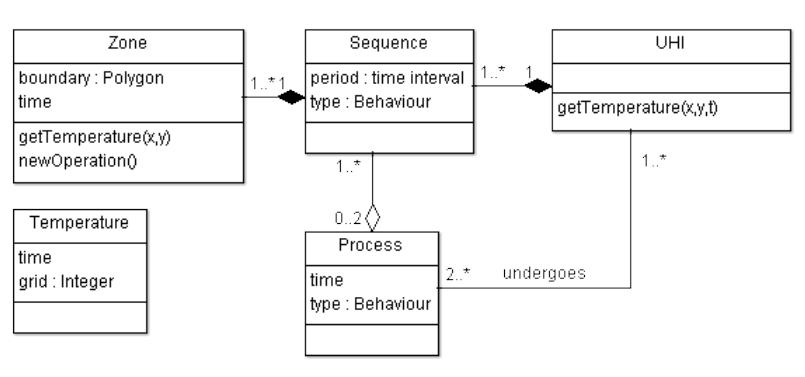

Figure 4: Class diagram of the UHI model.

\subsection{UHI behavior model}

UHI are extracted as sets of polygons such that temperatures within the polygons are higher than a reference rural temperature, which varies constantly through time. Hence, UHI are not modeled in a continuous time-space representation but by a composition of discrete objects from which the behavior is reconstructed. Adapting the framework proposed by (McIntosh and Yuan 2005) to the UHI behavior, we adopt the following definitions:

- an UHI zone is an area on a temperature image of contiguous cells whose values are above the threshold temperature;

- a sequence is a group of zones undergoing the same change in consecutive snapshots. Consecutive sequences can be related together by looking at the relationships between the last zone of a sequence and the first zone of the following sequence;

- a process is a group of sequences related by a topological transition. These sequences can belong to different UHI in the case of a split or a merge.

The life cycle of an UHI is then composed by a series of consecutive sequences and processes. Figure 4 presents the class diagram where zones, sequences and processes are objects that compose UHI. As sequences and processes are related, the life cycle of a UHI can be reconstructed from its processes. The temperature class is a discretization of the temperature defined by a $2 \mathrm{D}$ grid at each time instant. A zone is bounded by a polygon, having a finite extension, and defined at a given time instant. A sequence is then an aggregation of consecutive zones. Sequences are also linked to their previous and following sequences. This allows for the full qualitative description of the UHI behavior as a series of sequences where the UHI expands and contracts.

Processes model the topological transitions of Figure 4 One process is associated to one or several sequences, the role of a sequence depending on the type of process. For example, an inherited split is associated to the sequences of the main UHI preceding and following the split and to the sequences of the UHI splitting away from the main UHI. Contrary to (McIntosh and Yuan 2005), processes do not include only split and merge but all topological transitions, allowing the description of a UHI life cycle as a series of processes.

\section{APPLICATION TO UHI TRACKING}

\subsection{Study area}

The model was implemented and tested on a dataset acquired from the Southeast Coastal Ocean Observing Regional Association(SECOORA). The dataset provides air temperature data measured at approximately 1.5 meters above the ground from 171

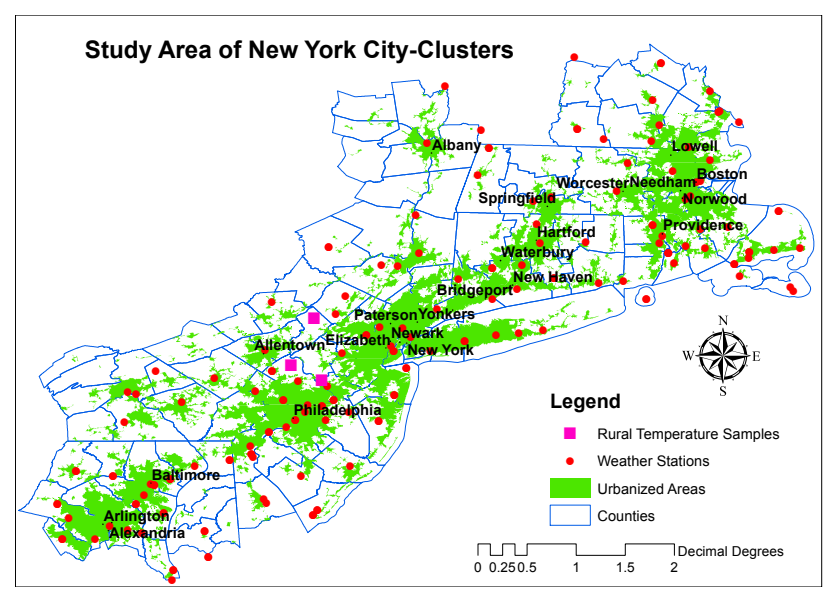

Figure 5: Urbanized areas in the northeast region of the USA.

weather stations along the coastline of New York representing a large urban area (Figure 5). Data were collected hourly from June 7, 2014 at 9.00 am to June 10, 2014 at $9.00 \mathrm{am}$. To have representative rural temperature for extracting zones of UHIs, three rural temperatures were collected and averaged each hour as the referenced rural temperature. Then, zones of UHIs were extracted automatically such that temperatures within which are higher than the referenced rural temperature. 102 sample points are located in the urbanized area, which ensures that a sufficient number of UHIs covering multiple urban areas can be generated. Temperatures over the whole area of study were interpolated by Universal Kriging, providing a temperature image at each time instant.

\subsection{Extraction of UHI changes}
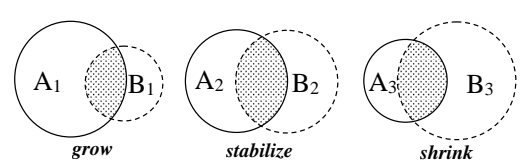

split
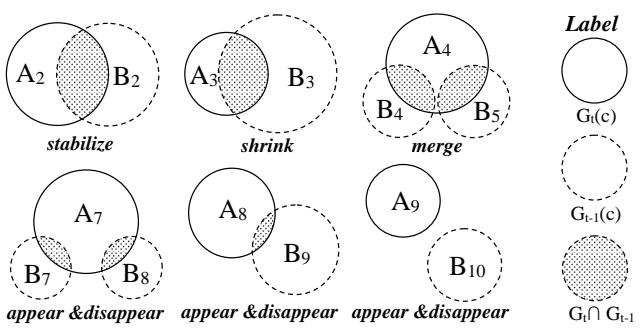

Figure 6: Intersection cases of the UHI $G_{t}(c)$ in two consecutive time instants

Behaviors shown in Figure 2 are identified for each time instant. Movements are computed based on the displacement and area variation as shown in Figure 6 If there is no intersection between two consecutive UHI polygons, it is reasonable to consider they have no relationship. If two polygons overlap on a sufficiently big area, they are considered to be two representations of the same UHI at two consecutive time instants. Hence changes at time $t_{i}$ are more specifically defined as follows:

- appear: the zone at time $t_{i}$ does not intersect with any zone at time $t_{i-1}$;

- disappear: the zone at time $t_{i-1}$ does not intersect with any zone at time $t_{i}$;

- expand: the UHI polygon intersects with a UHI at time $t_{i-1}$ and its area is bigger;

- contract: the UHI polygon intersects with a UHI at time $t_{i-1}$ and its area is smaller; 
- stable: the UHI intersects with a UHI at time $t_{i-1}$ and their areas are equivalent;

- merge: one UHI shape at $t_{i}$ overlaps with several UHI at $t_{i-1}$. If one UHI at $t_{i-1}$ is much bigger than the others and of an area close to the $\mathrm{UHI}$ at $t_{i}$, the $\mathrm{UHI}$ is supposed to be in the continuity of the big UHI and we have an inherited merge. If the new UHI cannot be associated to one specific $\mathrm{UHI}$, it is considered a new UHI qnd we have a generated merge.

- split: several UHI polygon at $t_{i}$ overlap with one polygon at $t_{i-1}$. If the shape at $t_{i}$ is similar to one specific polygon at $t_{i-1}$, it is an inherited split otherwise it is a generated split.

If two zones at $t_{i-1}$ overlap the same zone at $t_{i}$, three different situations may occur: (1) they merge together into one if both of them overlap significantly with the zone at $t_{i},(2)$ one disappears and the other one keeps existing when only one pair of zones respectively at $t_{t-1}$ and $t_{i}$ has a significant overlap, or (3) none of them have significant overlapping meaning that they are not related with each other such that the two at $t_{i-1}$ disappear and the one at $t_{i}$ appear.

\subsection{Computation}

The model was implemented and results from the tracking system were stored into an object-relational database managed with PostgreSQL 9.3.4. The database allows for the retrieval of processes and of the life cycle of each UHI through SQL queries.

Several threshold parameters were defined to recognize different types of movement and transformation. First, in order to be related together, zones identified at two consecutive time instants need to overlap significantly. This is done by checking if the intersection of two zones is similar in size to the smaller of the two zones. A relative threshold $\varepsilon_{\text {related }}$ is defined for this (equation 11.

$$
1-\frac{\operatorname{area}\left(U H I_{t_{i}} \cap U H I_{t_{i-1}}\right)}{\min \left(\operatorname{area}\left(U H I_{t_{i}}\right), \text { area }\left(U H I_{t_{i-1}}\right)\right)} \leq \varepsilon_{\text {related }}
$$

The type of event the UHI undergoes depends then on the number of associations a zone has with other zones. If a zone is associated with one single zone, no transformation occurs and the UHI is changing. If no association can be made, the UHI is appearing or disappearing. Finally, if several associations are made, a split or a merge is taking place.

Two UHI zones are considered of same size if their relative area difference is smaller than a threshold $\varepsilon_{\text {area }}$ (equation 2). Hence an UHI is considered to expand if its area has increased of $\varepsilon_{\text {area }}$ between two consecutive time instants. Similarly, if the UHI area decreases by $\varepsilon_{\text {area }}$, the UHI is contracting.

$$
\frac{\operatorname{area}\left(U H I_{t_{i}}\right)-\operatorname{area}\left(U H I_{t_{i-1}}\right)}{\operatorname{area}\left(U H I_{t_{i-1}}\right)} \leq \varepsilon_{\text {area }}
$$

A merge occurs when a zone at $t_{i}$ is associated with several zones at $t_{i-1}$. An inherited merge occurs if one of the new zones is significantly bigger than the others and is similar to the older zone. Hence, a UHI will undergo an inherited merge if one of its associated zones at $t_{i-1}$ has a similar shape as defined by equation 2 otherwise, all new zones correspond to new UHI and we have a generated merge.

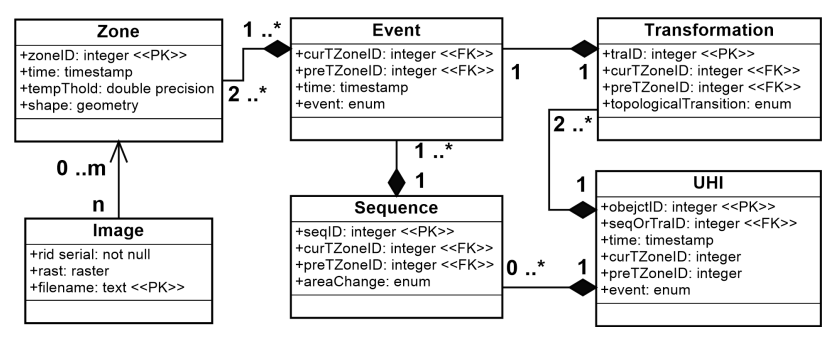

Figure 7: Database model

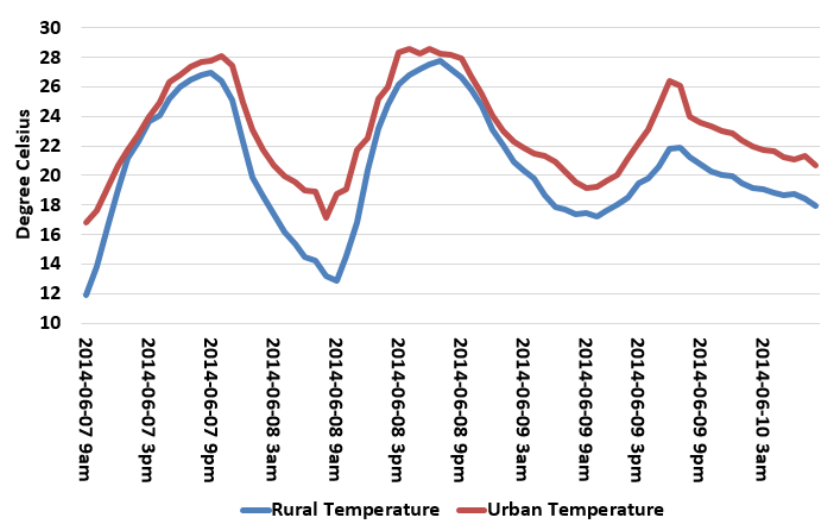

Figure 8: Database model

Similarly, a split occurs when a zone at $t_{i-1}$ is associated with several zones at $t_{i}$. An inherited split occurs if one of the older zones is significantly bigger than the others and is similar to the new zone. The difference between inherited and generated split is also checked with equation 2

\subsection{Logical model}

The logical model of the database (Figure 7 ) is derived from Figure 4 diagram. The Zone table is the main table used to derivate other tables. The boundary of the zone is defined by a polygon, which is extracted form the Image table storing the set of thermal images. The temperature threshold tempThold used to define the zone is also stored as an attribute as the images store the absolute air temperatures. In order to build up sequences and transitions, a new table Event was added storing the event computed for each zone. Hence this table stores not only the topological transformations but also the spatial changes of Figure 2. An Event instance is composed of two attributes which are the current and preceding zones. When the system has reconstructed all the events at each time, the Sequence table is defined relating zones belonging to the same sequence. The table of transformation Transformation is defined by relating topological transformations and sequences. Finally, the table UHI is built from the series of processes and sequences.

\section{RESULTS}

\subsection{Evolutionary trajectories of urban heat islands}

The threshold temperature is the average value of three sample points located in the rural area closely to the urban area (Figure 5. The threshold is computed at each time stamp and used for zone extraction. Figure 8 shows that the referenced rural temperature (the blue line) and the urban temperature in the core area of Philadelphia (the red line) have a periodical variation of 24 hours, and that the UHI phenomenon is obvious during the night when temperature difference is the largest. Thus, UHIs are constructed for each hour given the input parameters of $\varepsilon_{\text {related }}=0.35$ 


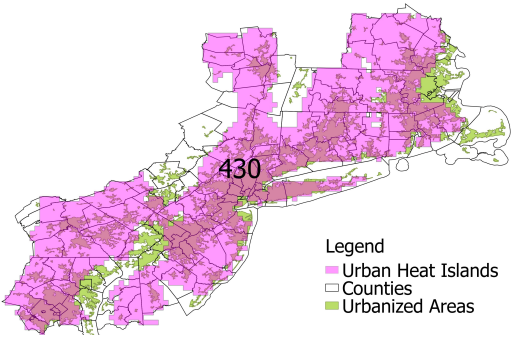

(a) $5 \mathrm{pm}$ : UHI covering the whole area.

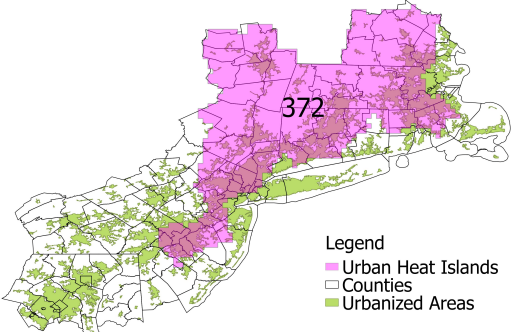

(d) 8pm: Inherited-merged into one UHI.

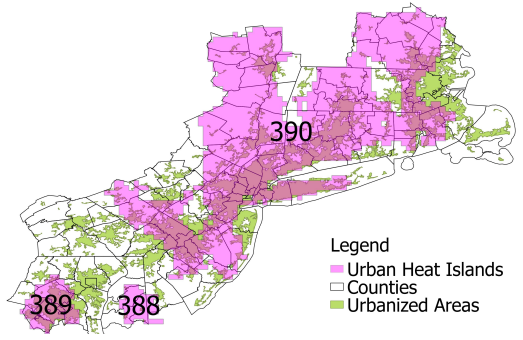

(b) 6pm: UHI split in three.

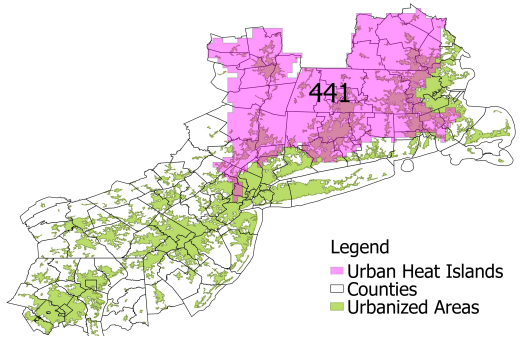

(e) $9 \mathrm{pm}$ : The UHI is contracting.

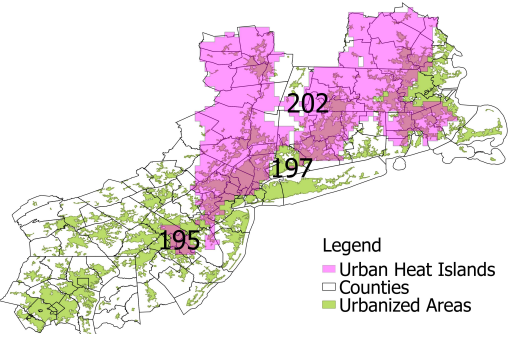

(c) 7pm: Three new UHIs appear.

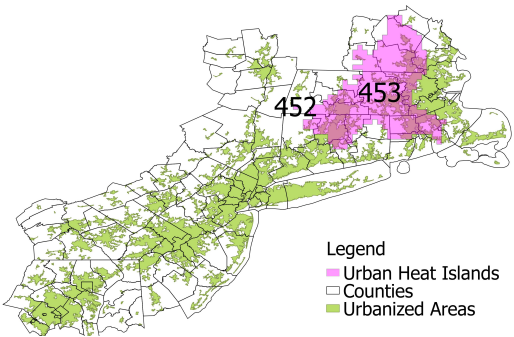

(f) 10pm: The small UHI disappears.

Figure 11: Evolution of an urban heat island at six consecutive time instants

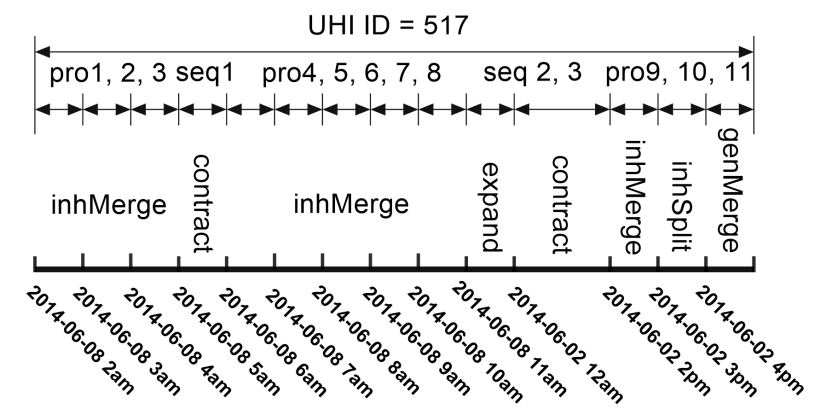

Figure 9: Database model

and $\varepsilon_{\text {area }}=0.1$. Directly extracting data from the tables, Figure 9 represents a complete life cycle of an UHI. The figure shows that this UHI appears from 2 am and continuously experienced three sequences and five processes, and finally disappeared due to the generated merge at $4 \mathrm{pm}$. This indicates that the system can effectively track the life cycle of each UHI.

Based on the achievement stated above, Figure 10 shows the spatiotemporal evolution of UHI based on an excerpt from the database. Firstly, an UHI identified as curTZoneId in the DBMS (i.e. 388 and 389) are inherited split (as different objects) and 390 is inherited split (as the same object) from 430, respectively. Then, three UHIs of 194, 195, and 197 are inherited merge (as different objects) with 202 (as the same object) as UHI 372. Subsequently, this UHI contracts as 411 , which is generated split as 452 and 453 simultaneously. Finally, these two UHIs disappear at $10 \mathrm{pm}$.

Figure 11 accordingly shows the spatial distribution of the above UHIs at six consecutive time instants, which mainly cover the New York city-clusters. The figure clearly indicates dynamic behaviors of UHIs during in a complete life-cycle from $5 \mathrm{pm}$ to 10 pm. More specifically, UHIs are continuously shrinking but do not disappear, possibly because the solar radiation flux decrease while anthropologic heat flux increases. Then, UHIs along the coastline of New York city disappear at $7 \mathrm{pm}$, probably benefiting from the cold sea-wind. Finally, the UHI over the Boston city-cluster disappears at $11 \mathrm{pm}$. Overall, this example shows that

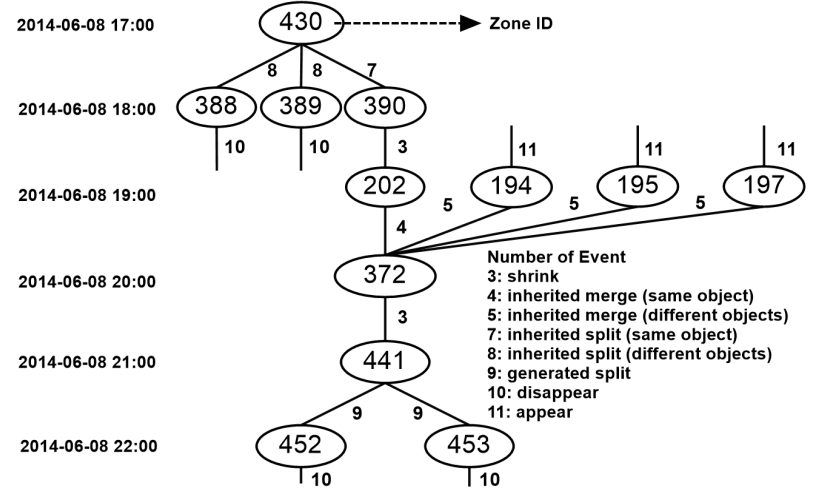

Figure 10: Evolutionary trajectories of urban heat islands drawn from part of the UHISequence table

the system can realistically track the evolution of the UHIs and qualify the different behaviors they go through.

\section{DISCUSSION AND CONCLUSION}

As an original contribution, this study conceptualizes UHI as a two-dimensional plus time field-object, proposes a model to describe the dynamic behaviors of UHIs, and develops a system to evaluate the performance of the model. The model provides a qualitative description of the evolution of a UHI through a series of sequences and processes.

The interpolated surface of near-ground air temperature is reliable and sufficient for constructing UHIs covering cities or cityclusters in a large area. In this work, rural temperature was set manually by choosing the temperature at several specific locations. However, when studying large areas, the rural temperature used as a reference may vary for the different city clusters. One perspective is to determine automatically the relevant rural temperature for each city cluster. To solve this problem, a strategy to automatically set temperature thresholds associated with time and space may be proposed.

Currently, only spatial changes are considered to describe continuous transformations in an UHI. Further work will consist in 
looking also at the evolution of the temperature within an UHI to provide also a thematic description. An objective is then to integrate in the model over environmental elements such as the land use or the buildings to study possible relationships and the influence they can have on the development of UHI. For that purpose, the UHI behavior may be represented at multiple levels as it can be influenced by factors at more local or global levels over different time scales.

\section{ACKNOWLEDGMENTS}

This research has been funded by 2013/14 General Research Fund of The Hong Kong Polytechnic University in the project of $D e$ velopment of a spatiotemporal data model for the analysis of the dynamic behaviour of urban heat islands (Project No. 515513), and Dr. Man Sing Wong also acknowledges the supports from the grant of Early Career Scheme, the Research Grants Council of Hong Kong (Project No. 25201614), and the grant G-YM85 from the Hong Kong Polytechnic University.

\section{REFERENCES}

Bothwell, J., Yuan, M., 2010. Apply concepts of fluid kinematics to represent continuous spacetime fields in temporal GIS. Annals of GIS, 16(1), 27-41.

Bothwell, J., Yuan, M., 2011. A Kinematics-based GIS Methodology to Represent and Analyze Spatiotemporal Patterns of Precipitation Change in IPCC A2 Scenario. ACM SIGSPATIAL GIS '11, 152-161.

Buyantuyev, A., Wu, J., 2010. Urban heat islands and landscape heterogeneity: linking spatiotemporal variations in surface temperatures to land-cover and socioeconomic patterns. Landscape Ecol 25(1):17-33.

Dousset, B., Gourmelon, F., 2003. Satellite multi-sensor data analysis of urban surface temperatures and landcover. ISPRS Journal of Photogrammetry \& Remote Sensing, 58, 43-54.

Goodchild M.F., Yuan M. and Cova T.J. 2007. Towards a general theory of geographic representation in GIS. Int. J. Geogr. Information Science 21(3):239-260.

Guilbert, E., Lin, H., 2007. A New Model for Cloud Tracking and Analysis on Satellite Images. Geoinformatica, 11(3), 287-309.

Keramitsoglou, I., Kiranoudis, C. T., Ceriola, G., Weng, Q., Rajasekar, U., 2011. Identification and analysis of urban surface temperature patterns in Greater Athens, Greece, using MODIS imagery. Remote Sensing of Environment, 115(12), 3080-3090.

Lo, C. P., Quattrochi, D. A., Luvall, J. C., 1997. Application of high-resolution thermal infrared remote sensing and GIS to assess the urban heat island effect. Int. J. Remote Sensing, 18(2): 287304.

McIntosh, J., Yuan, M., 2005. Assessing Similarity of Geographic Processes and Events. Transactions in GIS, 9(2), 223245.

Nixon, V., Hornsby, K. S., 2010. Using geolifespans to model dynamic geographic domains. International Journal of Geographical Information Science, 24(9), 1289-1308.

Renolen, A., 2000. Modelling the Real World: Conceptual Modelling in Spatiotemporal Information System Design. Transactions in GIS, 4(1), 23-42.
Stathopoulou, M., Cartalis, C., 2006. Daytime urban heat islands from Landsat ETM+ and Corine land cover data: An application to major cities in Greece. Solar Energy 81: 358-368.

Wong M. S., Nichol J. E., 2013. Spatial variability of frontal area index and its relationship with urban heat island intensity. International Journal of Remote Sensing, 34(3), 885-896.

Yuan M. and Hornsby K. 2008. Computation and Visualization for Understanding Dynamics in Geographic Domains. CRC Press. 